\title{
Diet quality in subjects with and without depressive and anxiety
}

disorders

Deborah Gibson-Smith1, d.gibson-smith@ggzingeest.nl

Mariska Bot ${ }^{1}$, Ingeborg Brouwer ${ }^{2}$, Marjolein Visser ${ }^{2}$, Brenda W.J.H. Penninx ${ }^{1}$

(1)Department of Psychiatry, Amsterdam Public Health Research Institute, VU University Medical Center, Amsterdam, The Netherlands

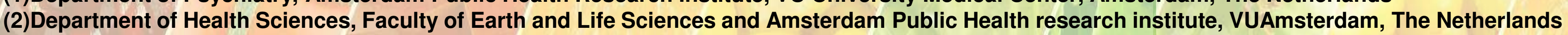

\section{Background}

Depressive symptoms have been associated with poorer diet quality(1), but diet quality studies among psychiatric samples are largely lacking.

\section{Objective}

This study will examine the association of depressive and anxiety disorders and their clinical characteristics (type of disorder, chronicity, and severity) with diet quality.

\section{Method}

Subjects: 1635 adults from Netherlands Study of Depression and Anxiety 9 yr follow-up. Outcome: Mediterranean diet score (MDS), and the Alternative healthy eating index (AHEI) derived from a 238-item semi-quantative food frequency questionnaire Determinants:-Disorder type: Depression and anxiety disorders were established with the Composite International Diagnostic Interview.

-Duration: Number of previous follow-up intervals with depression/anxiety (current disorder), Controls $=0$ episodes, remitted $=1$ episode.

-Severity: Inventory of Depressive Symptomatology (IDS) (depression),

Fear Questionnaire and the Beck Anxiety Inventory (BAI)(anxiety)

Covariates: age, gender, education, smoking status, physical activity and energy intake Statistical Analysis: Linear regression to estimate associations between (clinical characteristics of) depressive and anxiety disorders and standardized diet quality scores.

Disorder Type
Control (reference)
Remitted
Current depressive disorder (w/o anxiety)
Current anxiety disorders (w/o depression)
Current comorbidity
No of assessments with a disorders
Current with 0 -1 assessments/Control/Remitted
Current with 2 assessments
Current with 3 assessments
Current with 5 assessments
Severity
IDS Score
FAI Score
Fear Score
$\quad \mathbf{B}$ (95\% CI)

\section{Conclusion}

Diet quality is poorer in persons with depression and anxiety disorders, especially when diet quality is operationalized according to the Mediterranean diet. The more severe and chronic depression and anxiety symptoms were, the poorer diet quality. Prospective studies are needed to confirm the temporal relationship between depression and anxiety disorders and diet quality . 